\title{
Erratum: Monte Carlo study of Lefschetz thimble structure in one-dimensional Thirring model at finite density
}

\author{
Hirotsugu Fujii, ${ }^{a}$ Syo Kamata $^{b}$ and Yoshio Kikukawa ${ }^{a}$ \\ ${ }^{a}$ Institute of Physics, University of Tokyo, \\ Tokyo, 153-8092 Japan \\ ${ }^{b}$ Department of Physics, Rikkyo University, \\ Tokyo, 171-8501 Japan \\ E-mail: hfujii@phys.c.u-tokyo.ac.jp, skamata@rikkyo.ac.jp, \\ kikukawa@hep1.c.u-tokyo.ac.jp
}

ERRATUM TO: JHEP12(2015)125

ARXiv EPrint: 1509.09141

In [1] we studied the Lefschetz thimble integration method for the one-dimensional Thirring model numerically based on the HMC algorithm proposed in ref. [2], and concluded that the numerical integration by picking up just a single dominant thimble fails to reproduce the exact result in the crossover region for the smaller $\beta$, while it works for the larger $\beta$ (rather insensitively to the lattice size $L$ ).

However, the authors found a simple bug in implementing the HMC algorithm constrained on a thimble in this model, i.e., in solving eq. (3.3) in [1], which may cause some inaccuracy of our previous simulations. Therefore, we have performed all the simulations again with the fixed code. We find that our main conclusions remain unchanged.

In these simulations, we chose the same HMC parameters as those of the original setup in [1]: $t_{0}=-4, N_{t}=20, \Delta t=0.1,0.05 \leq \lambda \leq 0.1$ for the Runge-Kutta method and $\tau=0.5, N_{\tau}=10$ for the Molecular dynamics. We generated 1,000 configurations and estimated error bars using Jackknife method with the same bin-size, a bin per 20 configurations.

The results are presented in figures 4-10 and we comment the changes from the previous results. One can generally recognize that the central values of the observables are almost unchanged from the original ones, while the error bars become slightly smaller. We show 


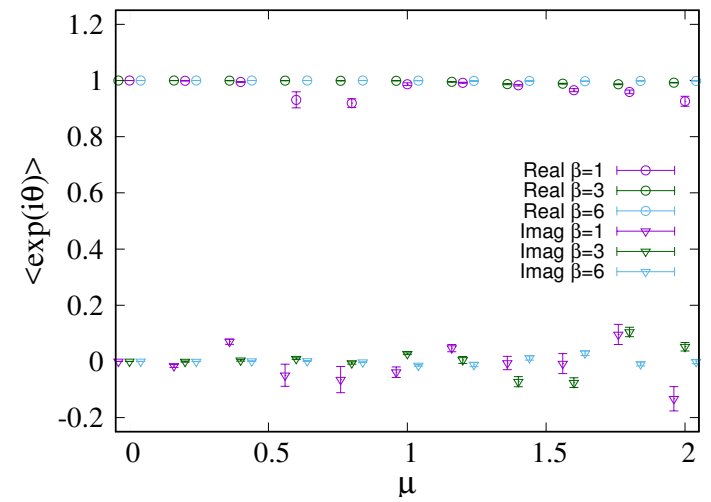

(a) $L=4$.

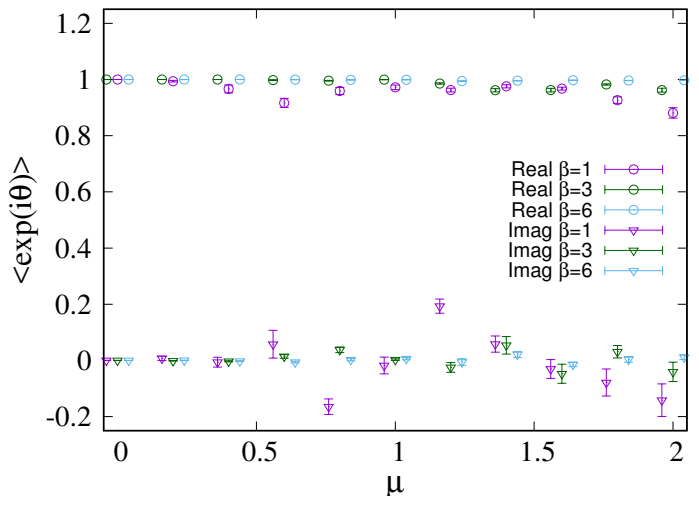

(b) $L=8$.

Figure 4. The averages of the residual phase factor for $m a=1, \beta=1,3,6$ and $L=4,8$.

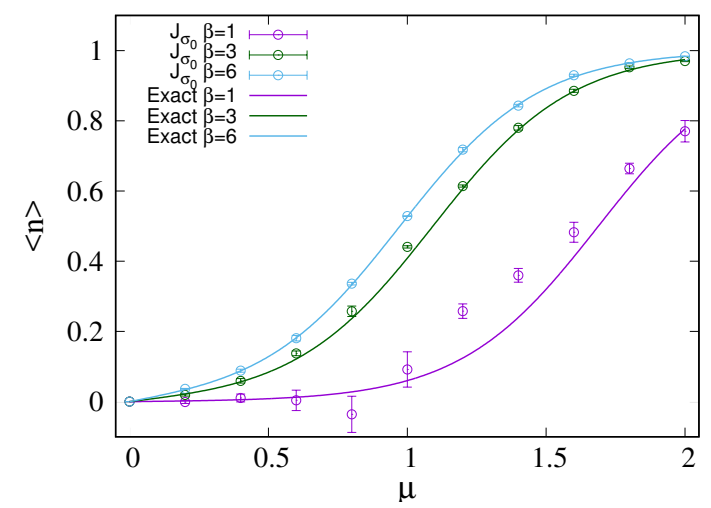

(a) Number density.

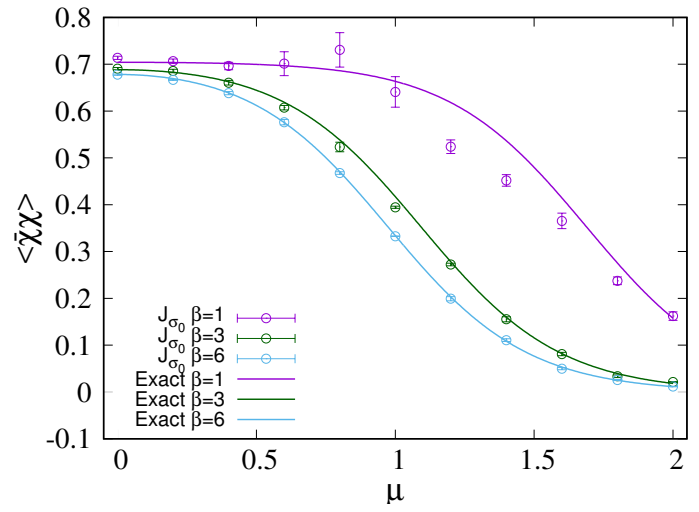

(b) Scalar condensate.

Figure 5. The number density and scalar condensate at $m a=1$ and $\beta=1,3,6$ on the lattice $L=4$.

the average residual phase in figure 4 . The real part behaves similarly to the previous one, being nearly consistent with unity, for all $L$ and $\beta$. But we notice some deviations from zero value in the imaginary part because of the reduced error bars, especially around the crossover region for $L=8$. We show the number density and scalar condensate in figures 6-8. The overall behavior is unaltered from the previous results, although the deviations from the exact solutions become slightly larger for $L=8, \mu=0.8$ and $\beta=3$. In the analysis of the continuum limit presented in figure 9 , the results at $L=8$ and $\mu / m=0.8$ becomes closer to the exact solutions, while the values at $\mu / m=1.0$ and $\mu / m=1.2$ for $L=8$ become slightly larger than the original ones in the number density and smaller in the scalar condensate. The agreement with the exact result is slightly improved for $L=16$ and $\mu / m=1.2$.

In summary, the changes from the previous results are quite minor, and therefore the conclusions in [1] are unchanged. 


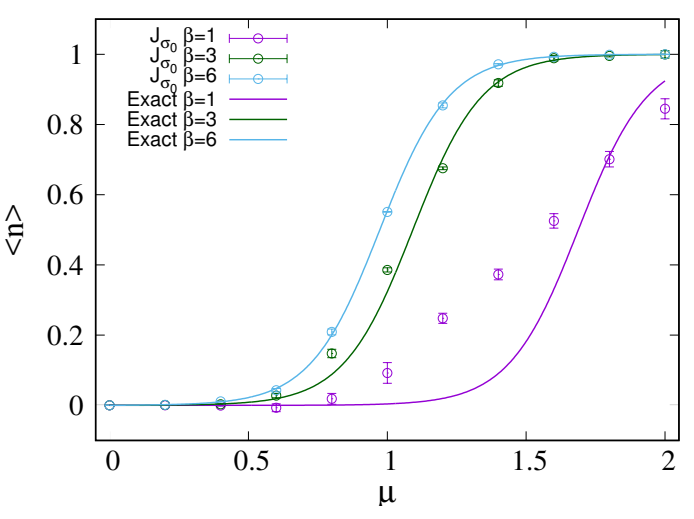

(a) Number density.

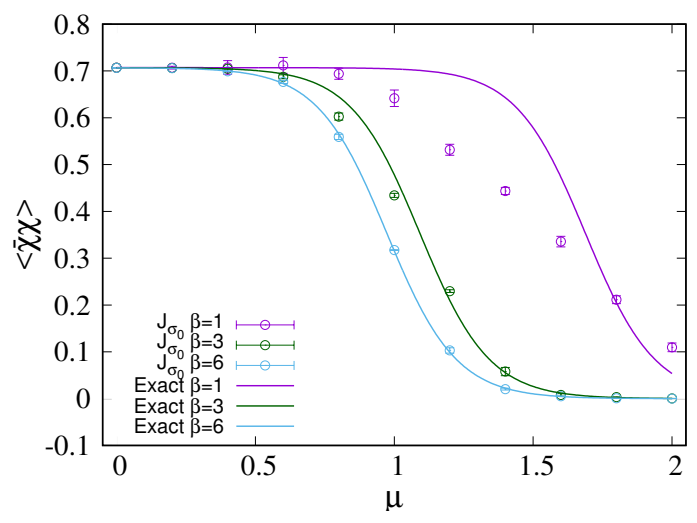

(b) Scalar condensate.

Figure 6. The number density and scalar condensate at $m a=1$ and $\beta=1,3,6$ on the lattice $L=8$.

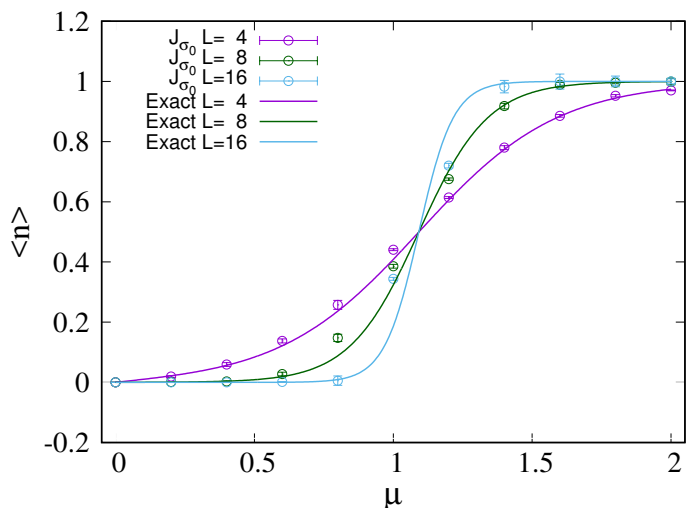

(a) Number density.

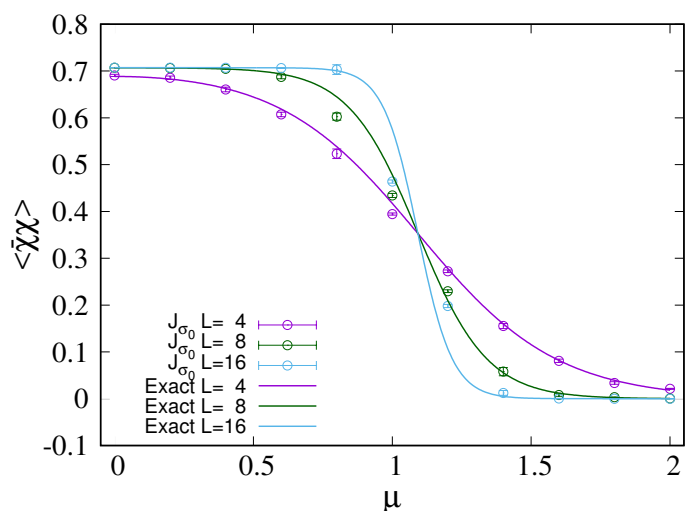

(b) Scalar condensate.

Figure 7. $L$-dependence of the number density and scalar condensate at $m a=1, \beta=3$.

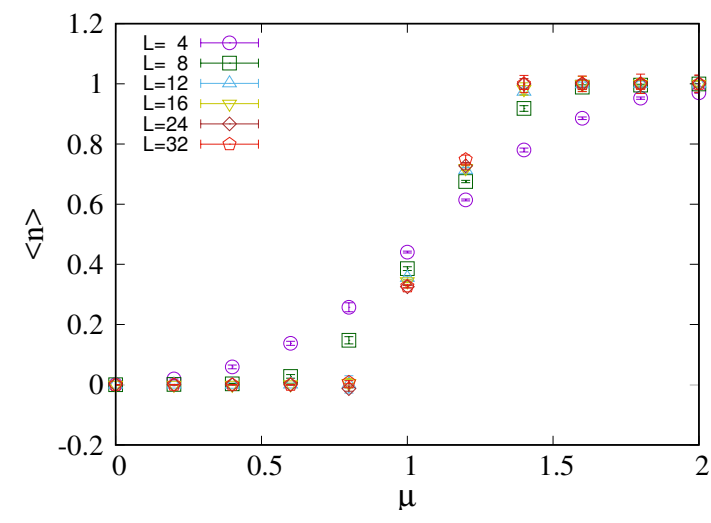

(a) Number density.

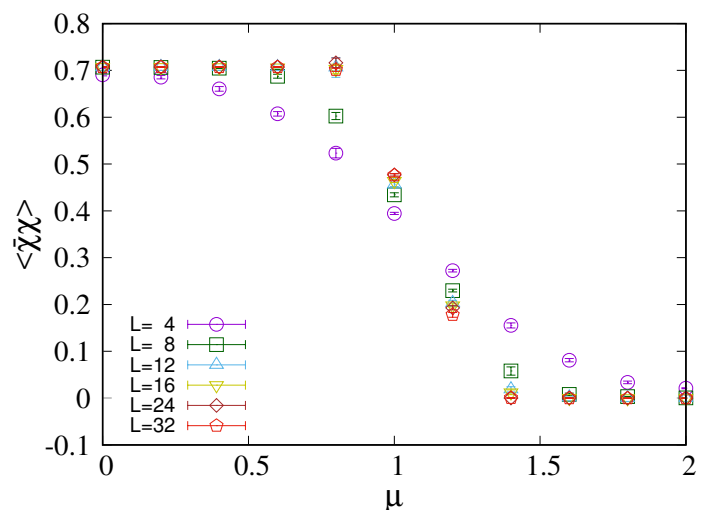

(b) Scalar condensate.

Figure 8. Low temperature limit of the number density and scalar condensate at $m a=1, \beta=3$. 


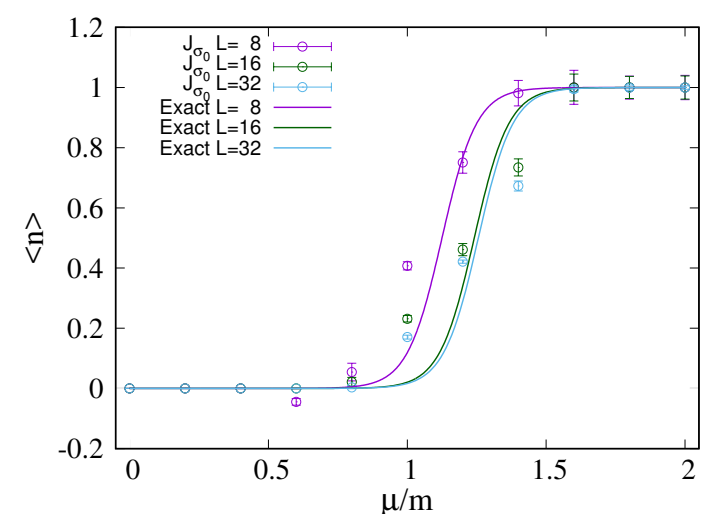

(a) Number density.

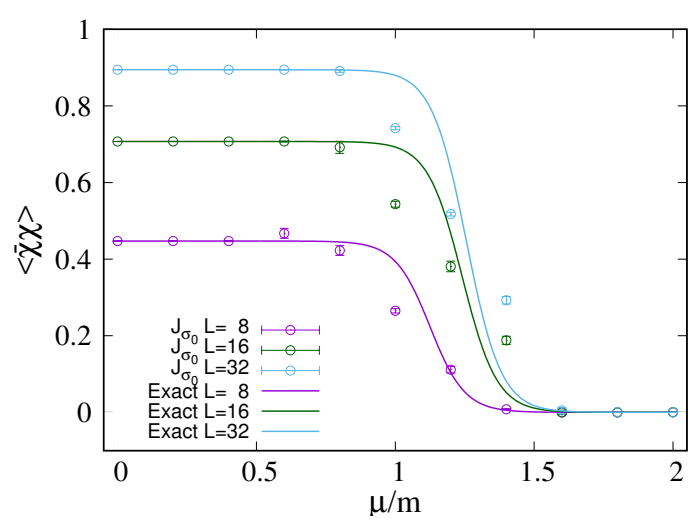

(b) Scalar condensate.

Figure 9. Continuum limit of the number density and scalar condensate at $L m=16$ and $\beta m=2$. We simulated with 8,16 and 32 lattice sites.

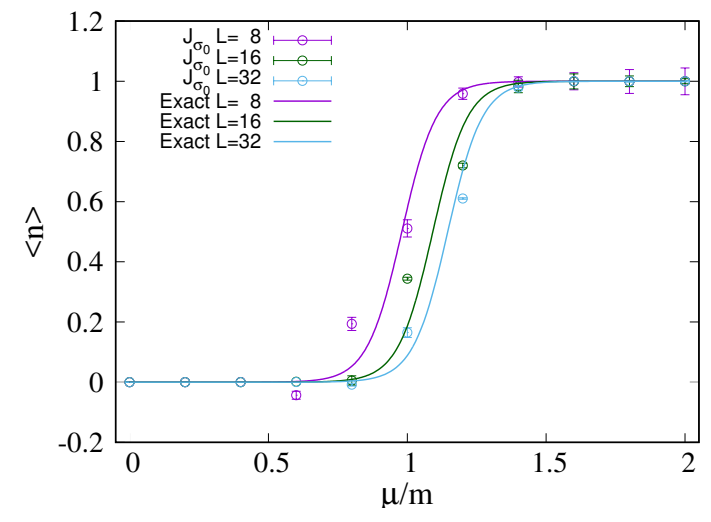

(a) Number density.

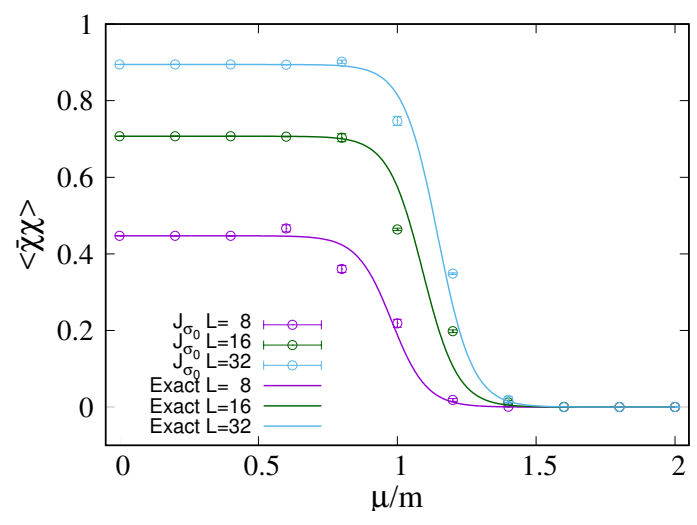

(b) Scalar condensate.

Figure 10. Continuum limit of the number density and scalar condensate at $L m=16$ and $\beta m=3$. We simulated with 8,16 and 32 lattice sites.

Open Access. This article is distributed under the terms of the Creative Commons Attribution License (CC-BY 4.0), which permits any use, distribution and reproduction in any medium, provided the original author(s) and source are credited.

\section{References}

[1] H. Fujii, S. Kamata and Y. Kikukawa, Monte Carlo study of Lefschetz thimble structure in one-dimensional Thirring model at finite density, JHEP 12 (2015) 125 [arXiv:1509.09141] [INSPIRE].

[2] H. Fujii, D. Honda, M. Kato, Y. Kikukawa, S. Komatsu and T. Sano, Hybrid Monte Carlo on Lefschetz thimbles - A study of the residual sign problem, JHEP 10 (2013) 147 [arXiv:1309.4371] [INSPIRE]. 